\title{
SHORT COMMUNICATION The Perceptual Grouping Criterion of Colinearity is Reflected by Anisotropies of Connections in the Primary Visual Cortex
}

\author{
Kerstin E. Schmidt, Rainer Goebel, Siegrid Löwel and Wolf Singer \\ Max-Planck-Institut für Hirnforschung, Deutschordenstrasse 46, 60528 Frankfurt am Main, Germany
}

Keywords: cat, area 17, tangential connections, orientation columns, elongation, strabismus

\begin{abstract}
An important step in the processing of visual patterns is the segmentation of the retinal image. Neuronal responses evoked by the contours of individual objects need to be identified and associated for further joint processing. These grouping operations are based on a number of Gestalt criteria. Here we report that connections in the visual cortex of the cat exhibit a highly significant anisotropy, preferentially linking neurons activated by contours that have similar orientation and are aligned colinearly. These anatomical data suggest a close relation between the perceptual grouping criterion of colinearity and the topology of tangential intracortical connections. We propose that tangential intracortical connections support perceptual grouping by modulating the saliency of distributed cortical responses in a context-dependent way. The present data are compatible with the hypothesis that the criteria for this grouping operation are determined by the architecture of the tangential connections.
\end{abstract}

Figure 1 illustrates that our visual system has the tendency to group contour segments that share the same orientation, especially if these are colinear (Field et al., 1993; Polat and Sagi, 1993, 1994; Kapadia et al., 1995). This implies that somewhere in the processing hierarchy responses to colinearly aligned contours must be distinguished from responses to the other, physically identical contours. The only cues for this distinction are the relations between the location and orientation of the surrounding contours. Therefore, selection of responses must occur in a context-dependent way at a level of processing where neurons have orientation-selective receptive fields. As neither the perceived location nor the general appearance of individual contour elements is influenced by context, the grouping operation must occur at a level where neurons have receptive fields whose size is equal to or smaller than that of the pattern elements and response selection must not alter the feature-selectivity of the neurons. These requirements make it likely that grouping according to the criterion of colinearity occurs at an early level of cortical processing. A possible substrate for context-dependent response selection could be long-ranging tangential connections among cells tuned to a particular stimulus orientation. These appear to be ideally suited to serve as a substrate for perceptual grouping: they (i) span large distances and hence can convey contextual information (Rockland and Lund, 1982; Gilbert and Wiesel, 1983; Martin and Whitteridge, 1984), (ii) selectively link neurons with similar feature preferences and hence could accomplish grouping according to criteria of similarity (Ts'o et al., 1986; Gilbert and Wiesel, 1989; Ts'o and Gilbert, 1989; Malach et al., 1993), (iii) modulate the amplitude of responses but not their feature-selectivity, and (iv) synchronize the responses of cortical neurons in a context-dependent way, and hence can selectively enhance the saliency of responses without interfering with their feature-specificity (Gray et al., 1989; Engel et al., 1991). If the tangential connections contribute to response selection in perceptual grouping their functional architecture should reflect the respective grouping rules. Grouping according to the criterion of colinearity could be achieved if neurons with colinearly aligned receptive fields were preferentially linked through reciprocal connections that enhance the saliency of responses.

In this study, we investigated whether the long-ranging tangential connections in area 17 of the cat exhibit the predicted tendency to preferentially interconnect neurons with colinearly aligned receptive fields. If so, tangential fibres linking neurons with vertically oriented receptive fields should be more numerous and extend further along trajectories that are parallel rather than orthogonal to the representation of the vertical meridian, while the reverse should be the case for connections between neurons with horizontally oriented receptive fields. Evidence for such an anisotropy of tangential connections has been reported for the primary visual cortex of tree shrews (Fitzpatrick, 1996).

We investigated one normally raised cat (AN) and four strabismic cats (A1-A4) in which divergent strabismus was surgically induced 

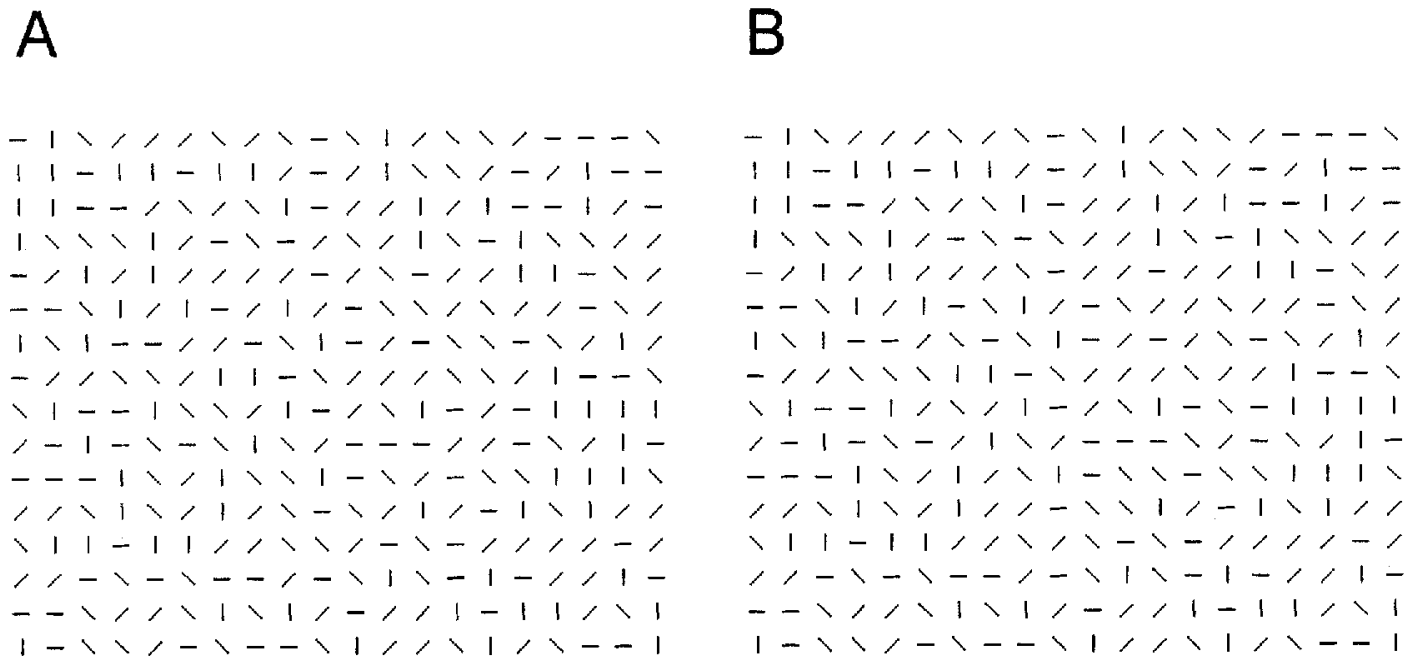

FIG. 1. An example of perceptual grouping on the basis of colinearity and vicinity. The colinearly arranged line segments in A that define the edges of a rhombus get grouped together and stand out from the randomly distributed line segments of the background. In B the line segments are arranged in the same way as in A except those that defined the outline of the rhombus, which are now parallel to each other and hence orthogonal to the corresponding segments in A.

at the age of 17 days. The strabismic cats had been the subjects of another investigation (Schmidt et al., 1994; K. E. Schmidt, D.-S. Kim, W. Singer, T. Bonhoeffer and S. Löwel, submitted for publication). At the age of 3 months, orientation preference maps were visualized by optical imaging of intrinsic signals (Grinvald et al., 1986) while the animals were stimulated monocularly with moving gratings of four orientations $\left(0^{\circ}, 45^{\circ}, 90^{\circ}, 135^{\circ}\right)$ with a spatial frequency of 0.5 cycles $/{ }^{\circ}$ and a drift velocity of $4 \%$. At the end of the optical recording session, rhodamine- and fluorescein-coupled latex microspheres (red and green beads; Luma Fluor Inc, Naples, FL; Katz et al., 1984; Katz and Iarovici, 1990) were pressure-injected into domains of unambiguous orientation and eye preference, as inferred from the optical activity maps. For subsequent correlation between the distribution of retrogradely labelled cells and orientation domains, territories showing the orientation preference of the injection site were additionally labelled using $\left[{ }^{14} \mathrm{C}\right] 2$-deoxyglucose autoradiography after monocular stimulation with horizontal or vertical gratings $\left(0.5 \mathrm{cycles}^{\circ}, 4^{\circ} / \mathrm{s}\right) 4-5$ days later. In the normal cat $(\mathrm{AN})$, the red and green tracers were injected in domains preferring horizontal and vertical contours respectively, and 2-deoxyglucose labelling was obtained by stimulation with horizontal contours. Retrogradely labelled neurons were then plotted in four to six flat-mounted sections from the supragranular layers of area 17. To investigate the topographical relationship between tangential connections and isoorientation domains and to relate the cell distributions to the visual field representation, the cell plots were superimposed on the 2deoxyglucose-labelled orientation domain map of the same section (for methods see Löwel and Singer, 1992). As a landmark for the representation of the vertical meridian we used the $17 / 18$ border, which is clearly visible in the 2-deoxyglucose patterns.

To quantify the anisotropies we computed polar plots of the distribution of retrogradely labelled cells around the centre of tracer injection for four representative sections with a resolution of $10^{\circ}$ (Fig. 3). The index $D$ of each data point reflects the number of labelled neurons at the respective angle $\pm 5^{\circ}$, and their distance from the centre of the injection site. For a particular angle $\phi$ this value is computed as $D_{\phi}=\Sigma d\left(C_{x, y} I_{x, y}\right)$ where $d$ is the Euclidean distance, $C_{x, y}$ represents the coordinates of a cell falling into the corresponding $10^{\circ}$ sector $\left(\phi \pm 5^{\circ}\right)$ and $I_{x, y}$ represents the coordinates of the injection site. The plots were scaled to the largest value of each distribution. In order to relate the polar plots to the coordinates of the visual field map, we determined the border between areas 17 and 18 from the 2deoxyglucose map, and defined the orientation parallel to this border as the 'vertical' orientation of the plots.

To obtain a quantitative measure of the elongation of the distribution of retrogradely labelled neurons, we used circular statistics (Batschelet, 1981). Labelled neurons contribute to the same axis of elongation if they are located in sectors that are radially symmetrical to the injection site. We were thus dealing with bimodal orientation data that had to be transformed for axial data analysis. Axial data are fully described by rotating a scanning axis through $180^{\circ}$ whereas vector descriptions require the full range of $360^{\circ}$. For this reason we included in the value $D_{\phi}$ at a particular angle $0^{\circ} \leqslant \phi<180^{\circ}$ both the cells located in sector $\phi \pm 5^{\circ}$ and the sector $180^{\circ}+\phi \pm 5^{\circ}$. To apply circular statistics these $180^{\circ}$ distributions were then stretched to the full turn of $360^{\circ}$. The result of these transformations is illustrated in the upper diagrams of Figure 4 based on the data presented in the lower diagrams of Figure 3. From the computed circular representations a section mean vector can be calculated whose length $(r)$ and direction $(\phi)$ characterize the form of the distribution. The vector is calculated trigonometrically by using the formula given for grouped data in Batschelet (1981), namely, for the length of the vector $(r)$ :

$$
r=\left[\left\{\left(\Sigma n_{i} \cdot \cos \phi_{i}\right) / \Sigma n_{i}\right\}^{2}+\left\{\left(\Sigma n_{i} \cdot \sin \phi_{i}\right) / \Sigma n_{i}\right\}^{2}\right]^{1 / 2}
$$

where $n_{i}$ represents the number of labelled neurons in each group of a $10^{\circ}$ sector and $\phi_{i}$ is the angle of the respective sector. The mean direction of this vector can then be calculated using the equation:

$$
\phi=\arctan \left[\left\{\left(\Sigma n_{i} \cdot \cos \phi_{i}\right) / \Sigma n_{i}\right\} /\left\{\left(\Sigma n_{i} \cdot \sin \phi_{i}\right) / \Sigma n_{i}\right\}\right]
$$

The length of the vector is a value between 0.0 and 1.0. A value of 0.0 would indicate a circular distribution with no elongation whereas a value of 1.0 would indicate that all cells fall into the same $10^{\circ}$ sector.

In order to test whether the distribution of labelled neurons for each section differed significantly from a uniform distribution we applied 
TABLE 1. Angles of the mean vectors based on all sections analysed for each of the six injection sites in five animals

\begin{tabular}{lllllr}
\hline Animal & Colour & $N$ & $n \pm$ SD & Orientation & $\phi$ \\
\hline AN & green & 5 & $744 \pm 210.9$ & vertical & $106^{\circ}$ \\
AN & red & 5 & $148 \pm 106.4$ & horizontal & $11^{\circ}$ \\
A1 & green & 5 & $286 \pm 10.2$ & vertical & $96^{\circ}$ \\
A1 & red & 4 & $304 \pm 4.8$ & vertical & $89^{\circ}$ \\
A2 & green & 5 & $352 \pm 8.8$ & horizontal & $17^{\circ}$ \\
A2 & red & 5 & $79 \pm 4.6$ & horizontal & $43^{\circ}$ \\
A3 & green & 4 & $236 \pm 6.5$ & horizontal & $130^{\circ}$ \\
A4 & red & 5 & $130 \pm 6.5$ & vertical & $87^{\circ}$ \\
\hline
\end{tabular}

Colour, colour of injected latex microspheres; $N$, number of sections; $n$, mean number of labelled neurons; SD, standard deviation; orientation, preferred stimulus orientation of the neurons at the injection site; $\phi$, orientation of the injection mean vectors.

the Rayleigh test for directionality (Batschelet, 1981). The test statistic (z) is expressed as $z=n r^{2}$, where $n$ represents the number of all labelled cells in a section. This test indicated that the distributions of almost all sections $(36 / 38)$ were significantly modulated $(P<0.05)$. The lower diagrams of Figure 4 show the section mean vectors of distributions for five sections of the normally reared cat grouped according to injections in columns of vertical (left diagram) and horizontal orientation preference (right diagram). The large arrow represents the mean vector for all cells labelled from a particular injection and was computed from the mean vectors of individual sections. The angles of the mean vectors for all injections and the cell numbers on which the respective calculations are based are listed in Table 1.

The vector length can be used to calculate an angular deviation $(s)$ according to the equation $s=180^{\circ} / \pi[2(1-r)]^{1 / 2}$. Based on the computed angular deviation values, the Watson-Williams two-sample statistic $F=g(n-2)\left(s^{2} / \bar{s}^{2}-1\right)$ can be used to determine the significance of differences ( $s^{2}$ is the angular variance of the combined sample, $\bar{s}^{2}$ is the average of the angular variances for the two samples and $g$ is a constant; Batschelet, 1981). This test showed that the section mean angles obtained after injections in columns with vertical and horizontal orientation preference differed highly significantly from one another $\left(F_{1,36}=12.67, P<0.01\right)$.

In all cases (five hemispheres, seven injections), retrogradely labelled cells were grouped in clusters located mainly within domains that had the same orientation preference and ocular dominance as the injection site (Fig. 2). Following monocular stimulation with a single orientation, the zones labelled with 2-deoxyglucose contained $58 \pm 5 \%$ of the labelled cells $\left(P<0.0005, \chi^{2}\right.$ test $)$ but occupied only $30 \%$ of the cortical surface (Schmidt et al., 1994; K. E. Schmidt, D.-S. Kim, W. Singer, T. Bonhoeffer and S. Löwel, submitted for publication). Comparison of panels $\mathrm{A}$ and $\mathrm{B}$ in Figure 2 suggests that fibres connecting neurons with vertically oriented receptive fields (Fig. 2A) are more numerous and extend further in the anterioposterior than in the mediolateral direction, while the reverse seems to be the case for fibres connecting neurons with horizontally oriented receptive fields (Fig. 2B). This is also reflected by the corresponding polar plots. Data points with the largest distance from the centre of the injection site are preferentially located at angles around 100 or $280^{\circ}$ in Figure 3A (upper and lower plots) and around 15 or $210^{\circ}$ in Figure 3B (upper and lower plots). Although a considerable number of connections extend in the respective orthogonal directions, in particular in Figure $3 \mathrm{~B}$ at $270^{\circ}$ (upper plot) and at $130^{\circ}$ (lower plot), all distributions were asymmetrical, exhibiting significant elongation along axes that were either parallel or orthogonal to the representation of the vertical meridian. As demonstrated in the lower part of Figure 4 , the injection mean vector is around $100^{\circ}$ for the green injection in cat $\mathrm{AN}$, which had been placed in a column preferring vertical orientations, and around $10^{\circ}$ for the red injection, which had been placed in a column preferring horizontal contours. This confirms that tangential connections linking neurons with vertically oriented receptive fields are more numerous and extend further along trajectories parallel to the vertical meridian while the reverse is true for fibres connecting columns preferring horizontal orientations.
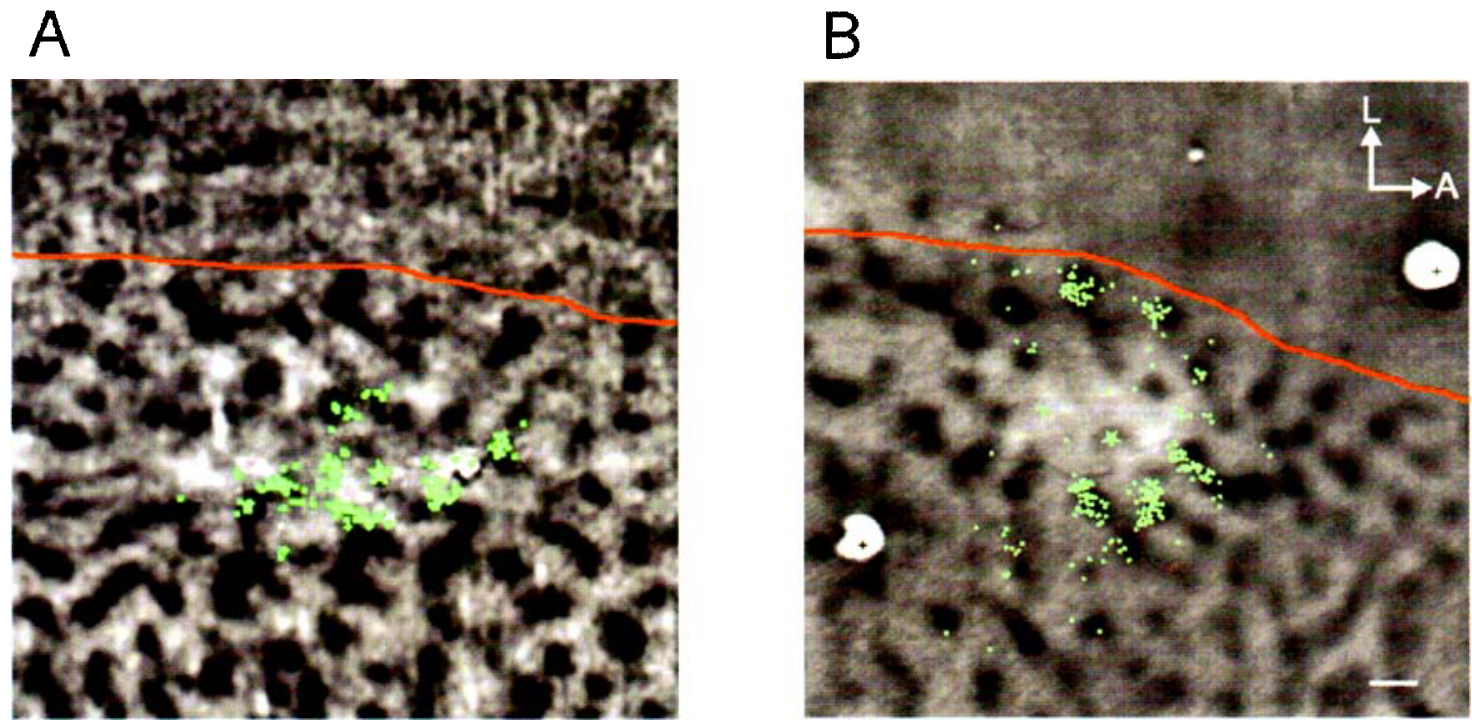

FIG. 2. Autoradiographs of supragranular flat-mounted sections demonstrating the layout of 2-deoxyglucose-labelled monocular orientation domains of two strabismic cats whose right eyes had been stimulated with moving vertical (cat A1, A) or horizontal (cat A2, B) bars. Because of the selected stimulus parameters, only columns in area 17 are labelled. The continuous line represents the $17 / 18$ border, i.e. the representation of the vertical meridian. The data represent retrogradely labelled neurons in the same sections that produced the respective autoradiographs. Labelled neurons are indicated by dots (A, $n=145 ; \mathrm{B}, n=260$ ), injection sites by triangles. Note that cell distributions lie in register with the pattern of iso-orientation columns and are elongated along the vertical (A) or horizontal (B) cortical axis.. A, anterior; L, lateral. Scale bar, $1 \mathrm{~mm}$. 

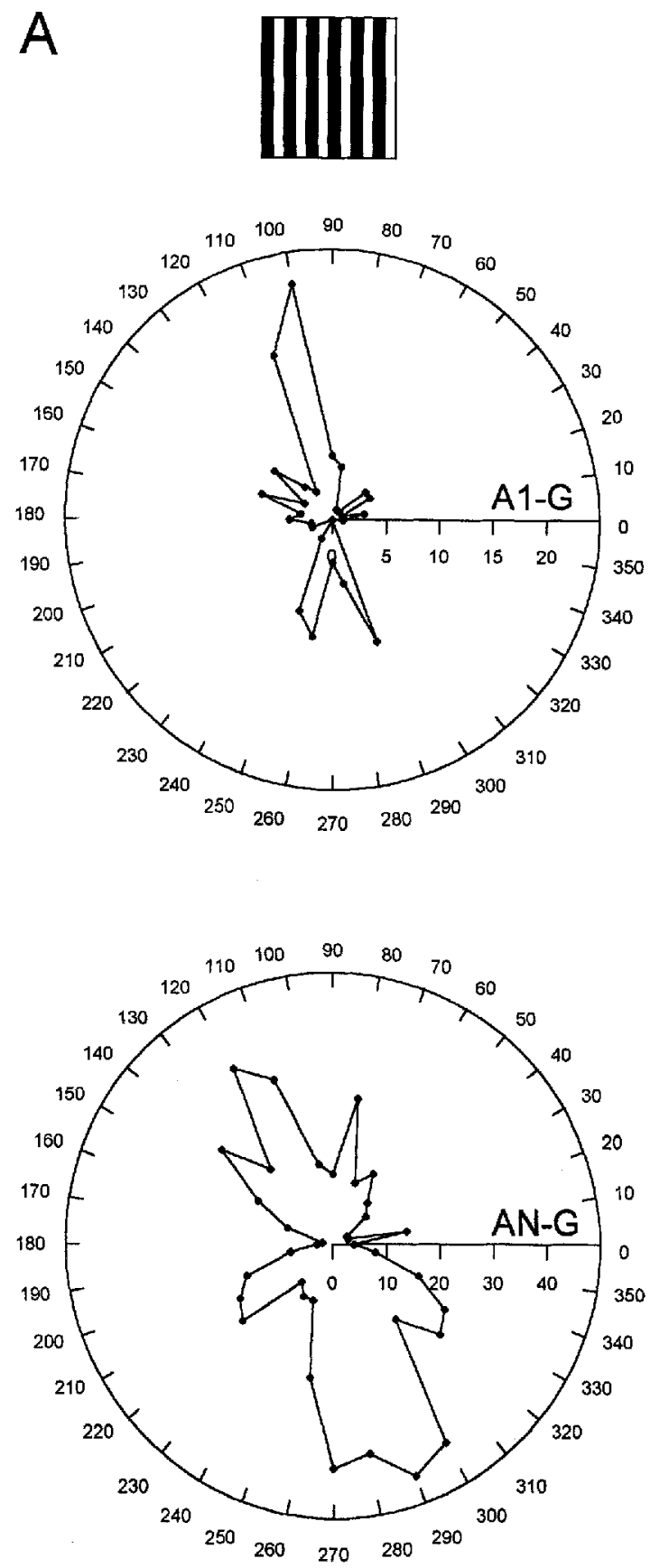
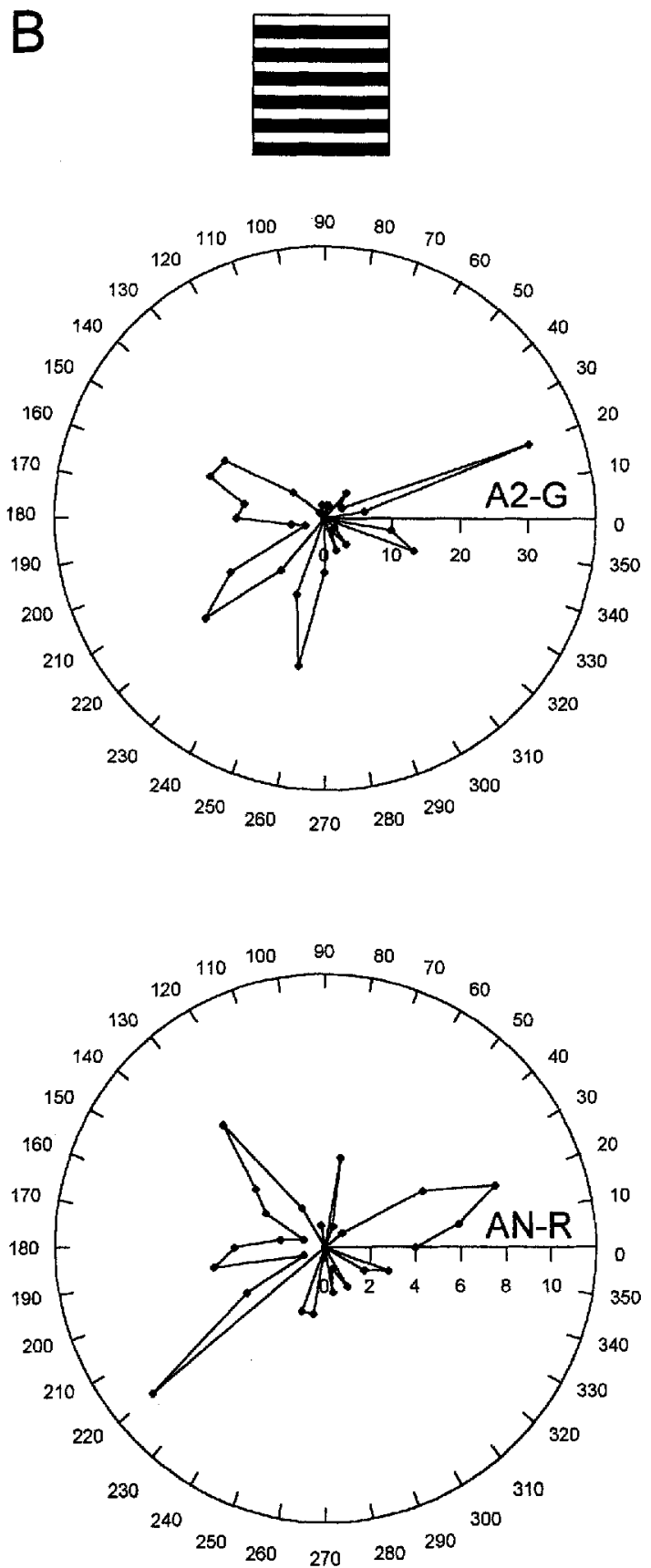

FIG. 3. Polar plots showing the distribution of retrogradely labelled cells in $10^{\circ}$ increments around the centre of tracer injection. Angles are adjusted according to the orientation of the respective $17 / 18$ border (at $0^{\circ}$; see text). The distance of each point from the centre indicates the number and distance of labelled cells within the respective segment. The gratings used as stimuli to identify the injected orientations columns are demonstrated above the plots. The upper plots in A and B are based on the data depicted in Figure 2. The lower plots correspond to representative sections of cat AN, AN-G reflecting the green injection into a column preferring vertical contours, and AN-R the red injection into a neighbouring horizontal orientation column. Note that maximal elongations are rather orthogonal to each other. The lobular structure of the plots reflects the clustering of labelled neurons.

There is one source of artefact that could have caused anisotropies of the density distributions. All injections were close to the $17 / 18$ border because this region is accessible for optical recording. Since only labelled cells located within area 17 were used for the calculation of density distributions, the vicinity of the $17 / 18$ border lateral to the injection site could have resulted in artificial under-representation of connections with mediolateral trajectories. This was indeed the case for injection A3 (green), which was placed very close to area 18 , and explains the low elongation indices for this injection. However, in spite of this bias all injections into columns tuned to horizontal orientations (A2, A3, AN red) still led to distributions that were elongated along an axis parallel to the representation of the horizontal meridian. The injections in columns preferring vertical contours were all sufficiently far from the vertical meridian so that no retrogradely labelled cells had to be excluded from the plots (Fig. 2A).

Furthermore, one has to take into account that the $17 / 18$ border 

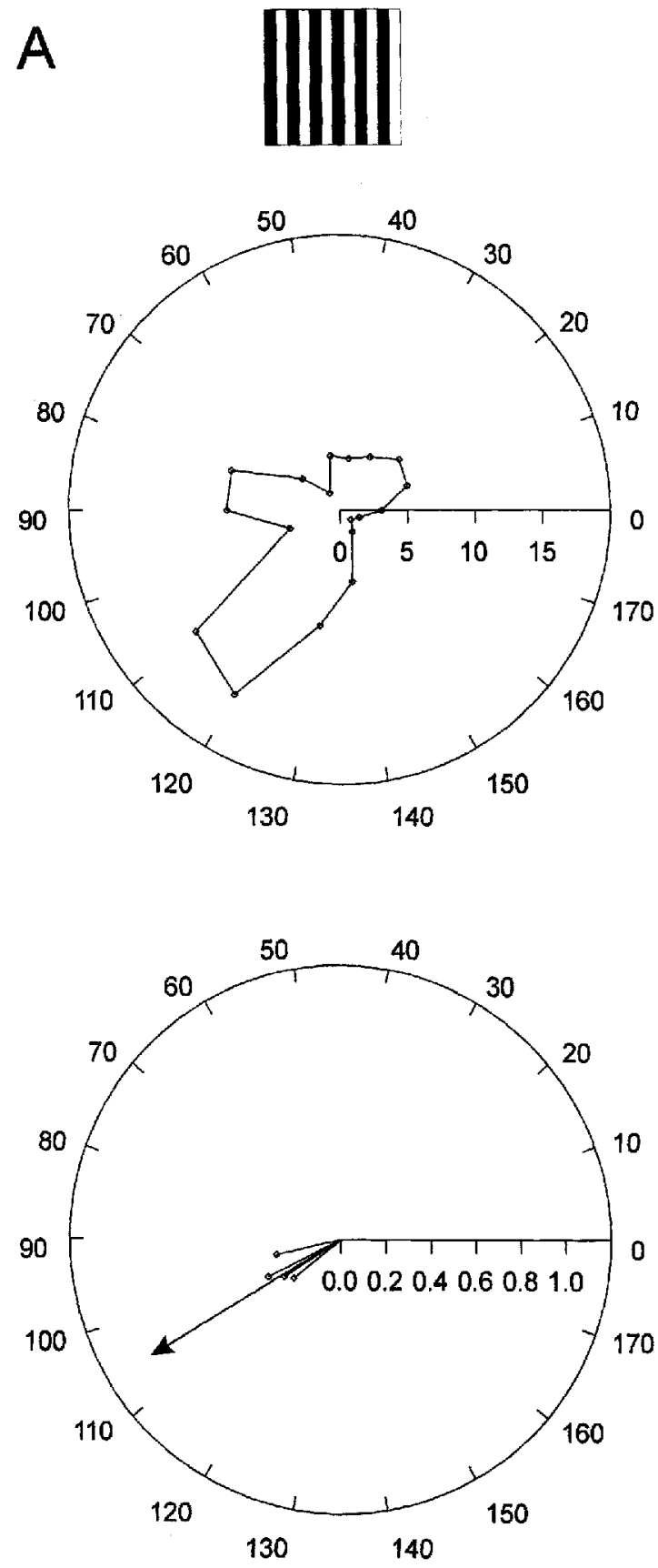
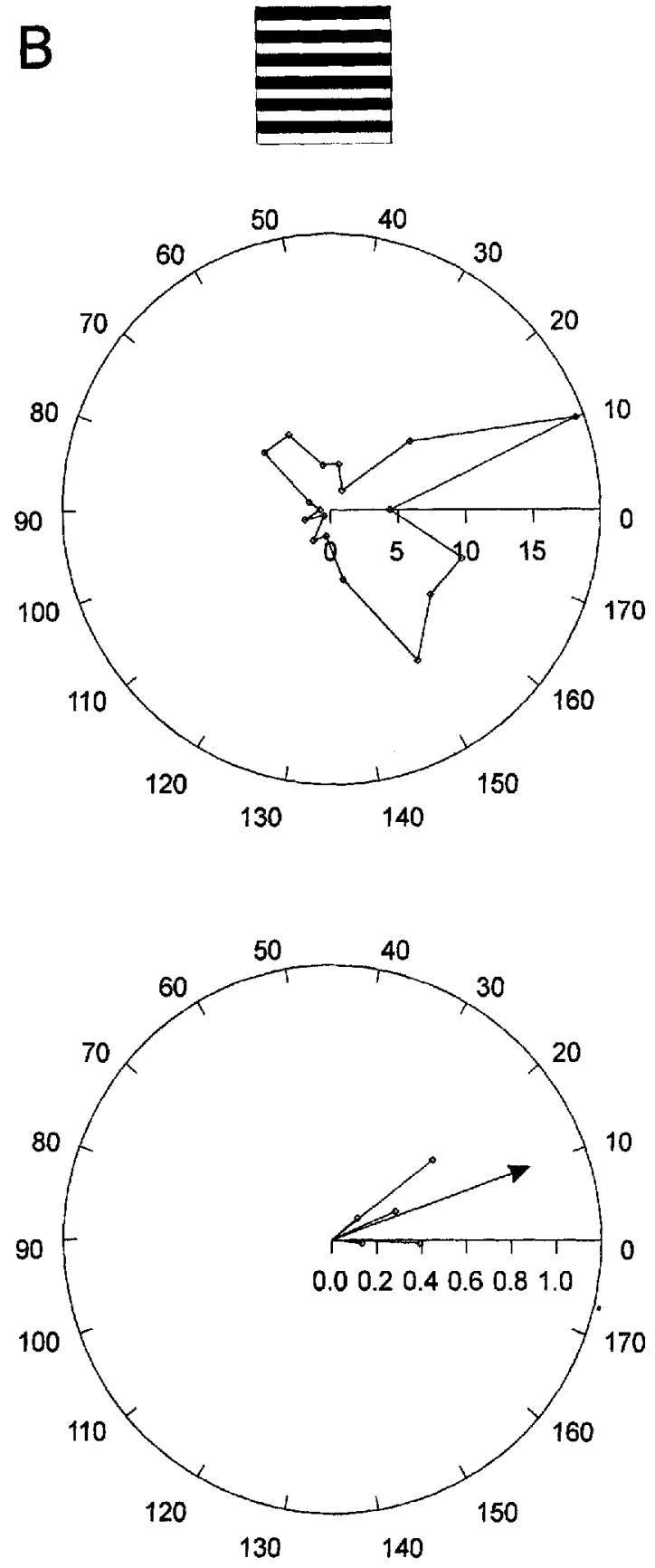

FIG. 4. Upper two plots show the distribution of labelled neurons in the different directions reduced to the range of $180^{\circ}$. Data in the top diagrams are

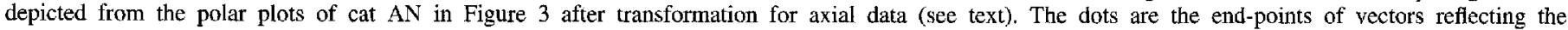

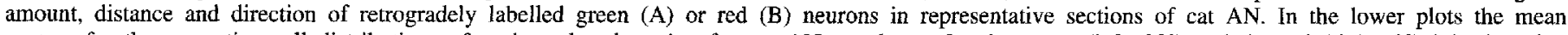
vectors for the respective cell distributions of each analysed section for cat AN are drawn for the green (left, $90^{\circ}$ ) and the red (right, $0^{\circ}$ ) injection site.

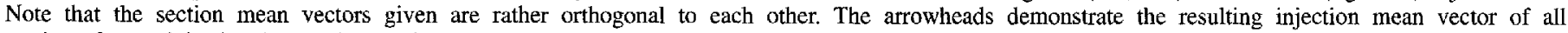
sections for an injection in a column of neurons preferring vertical (bottom left) or horizontal (bottom right) contours of cat AN.

was determined on autoradiographs of tangential sections that do not allow a precision of $1^{\circ}$. Since for cat AN, where both injection types were located in one animal, the difference between the maximal elongations of the polar plots (Fig. 3, lower plots) is close to $90^{\circ}$, small alterations from the defined vertical and horizontal axes could be based on a systematic failure to exactly determine the $17 / 18$ border.

Our results indicate that neurons whose receptive fields are tuned to the same orientation and are aligned colinearly are interconnected more frequently and over longer distances than neurons that share only the same orientation preference, the latter still being more connected than cells preferring different orientations. We consider it unlikely that the orientation-dependent anisotropy of the tangential connections is caused by strabismus for two reason: first, the normal cat gave the same results as the four squinting cats and second, squint leads to a relative reduction of neurons preferring vertical orientations (Singer et al., 1979) and to reduced spatial resolution for vertical gratings in the amblyopic eye (Sireteanu and Singer, 1980), but unless there is amblyopia, which is a rare event with divergent strabismus, 
all other monocular visual functions appear to be perfectly normal (Hubel and Wiesel, 1965; Freeman and Tsumoto, 1983; Freeman et al., 1983; Kalil et al., 1984; Sireteanu and Best, 1992). Thus, if squint had influenced the orientation-dependent bias in the distribution of tangential connections it should have caused a reduction of connections among neurons preferring vertical orientations. This should have attenuated rather than accentuated the anisotropy in the distributions following injections into vertical columns, and hence should have reduced the differences between the distribution of fibres linking vertical and horizontal columns respectively.

The present data are complementary to results of studies in the visual cortices of tree shrews (Fitzpatrick, 1996) and squirrel monkeys (Sincich and Blasdel, 1995), in which biocytin-labelled axon terminal fields were found to be elongated along the cortical representation of the labelled neuron's preferred stimulus orientation. Asymmetrical axonal fields and elongated distributions of retrogradely labelled neurons have also been described in the cat visual cortex (Kisvárday and Eysel, 1992), but it was concluded that the distributions are skewed both along and perpendicular to the representation of the neurons receptive field axis (Gilbert and Wiesel, 1983, 1989).

The highly significant anisotropies of tangential connections revealed in the present study suggest particularly strong interactions among neurons that prefer similar orientations and have colinearly aligned receptive fields. Several of the functions attributed to tangential connections could require such asymmetrical topographies. (i) Binding of coherently moving pattern elements according to the Gestalt criterion of common fate would require connections between neurons with similar direction preference, the receptive fields of which are located along the motion trajectory. This predicts connections between neurons with similar orientation preference along a cortical axis orthogonal to the receptive field's orientation preference. (ii) Endstopping could be achieved by tangential connections which extend predominantly between columns preferring colinear orientations as identified in this study, provided that these connections terminate on local inhibitory interneurons, but other explanations have also been proposed (Bolz and Gilbert, 1986). (iii) Phenomena such as length summation (Gilbert, 1977; Mitchison and Crick, 1982; Born and Tootell, 1991; Hammond and Fothergill, 1994), the creation of illusory contours between colinearly arranged contrast borders (Kanizsa, 1979) and the perceptual grouping of colinear line segments would require anisotropies of tangential connections similar to those identified in this study. The latter possibility agrees with the hypothesis that the tangential connections serve to select and associate in a contextdependent way the responses of feature-selective neurons for further joint processing, and that the criteria for this grouping operation reside in the architecture of these connections (Mitchison and Crick, 1982; Singer, 1995). The idea is that the tangential connections mediate cooperative interactions between selected groups of neurons and raise the saliency of the selected responses by increasing their rate and/or by making them synchronous. Both possibilities are supported by data. Responses to optimally oriented stimuli get enhanced when colinearly aligned contours are presented outside the classical receptive field (Nelson and Frost, 1985; Kapadia et al., 1995). Corticocortical connections have been shown to contribute to the synchronization of responses (Engel et al., 1991; Munk et al., 1995), and cells with colinearly arranged receptive fields synchronize their responses when activated with colinearly aligned line segments (Gray et al., 1989). Since during early development the architecture of tangential connections is shaped by neuronal activity (Callaway and Katz, 1991; Luhmann et al., 1991; Löwel and Singer, 1992), the possibility needs to be considered that the observed anisotropies are the result of visual experience. This would have the attractive implication that some of the Gestalt criteria for perceptual grouping are actually acquired by learning. As the effects of early strabismus show, tangential connections get removed between neurons whose activity is often uncorrelated, suggesting that they stabilize selectively between neurons that are frequently coactivated. Of all possible constellations of neurons, those with colinearly aligned receptive fields have the highest probability of getting coactivated because most contrast borders in natural scenes are elongated and, at least over short distances, are straight. Thus, the grouping criterion of colinearity could actually be extracted by a learning process that manifests itself by changes in the architecture of intracortical connections. It will be interesting to test this possibility by manipulating early visual experience and to see whether this applies also to other Gestalt criteria.

\section{Acknowledgements}

We thank D.-S. Kim for help in some of the experiments, E. Raulf and M. Sum for excellent technical assistance, M. Stephan for programs and the staff of the animal house of our Institute for excellent animal care.

\section{References}

Batschelet, E. (1981) Circular Statistics in Biology. Academic Press, London. Bonhoeffer, T. and Grinvald, A. (1993) The layout of iso-orientation domains in area 18 of cat visual cortex: optical imaging reveals a pinwheel-like organization. J. Neurosci., 13, 4157-4180.

Born, R. T. and Tootell, R. B. H. (1991) Single-unit and 2-deoxyglucose studies of side inhibition in macaque striate cortex. Proc. Natl Acad. Sci. USA, 88, 7071-7075.

Callaway, E. M. and Katz, L. C. (1991) Effects of binocular deprivation on the development of clustered horizontal connections in cat striate cortex. Proc. Natl Acad. Sci. USA, 88, 745-749.

Engel, A. K., König, P., Kreiter, A. K. and Singer, W. (1991) Interhemispheric synchronization of oscillatory neuronal responses in cat visual cortex. Proc. Natl Acad. Sci. USA, 88, 9136-9140.

Field, A. J., Hayes, A. and Hess, A. F. (1993) Contour integration by the human visual system: evidence for a local 'association field'. Vision Res., 33, 171-193.

Fitzpatrick, D. (1996) The functional organization of local circuits in visual cortex: insights from the study of tree shrew striate cortex. Cerebral Cortex, 6, 329-341.

Freeman, R. D., Sclar, G. and Ohzawa, I. (1983) An electrophysiological comparison of convergent and divergent strabismus in the cat: visual evoked potentials. J. Neurphysiol., 49, 227-237.

Freeman, R. D. and Tsumoto, T. (1983) An electrophysiological comparison of convergent and divergent strabismus in the cat: electrical and visual activation of single cortical cells. J. Neurophysiol., 49, 238-253.

Gilbert, C. D. (1977) Laminar differences in receptive field properties of cells in cat primary visual cortex. J. Physiol. (Lond.), 268, 391-421.

Gilbert, C. D. and Wiesel, T. N. (1983) Clustered intrinsic connections in cat visual cortex. $J$. Neurosci., 3, 1116-1133.

Gilbert, C. D. and Wiesel, T. N. (1989) Columnar specificity of intrinsic horizontal connections in cat visual cortex. J. Neurosci., 9, 2432-2442.

Gray, C. M., König, P., Engel, A. K. and Singer, W. (1989) Oscillatory responses in cat visual cortex exhibit inter-columnar synchronization which reflects global stimulus properties. Nature, 338, 334-337.

Grinvald, A., Lieke, E., Frostig, R. D., Gilbert, C. D. and Wiesel, T. N. (1986) Functional architecture of cortex revealed by optical imaging of intrinsic signals. Nature, 324, 361-364.

Hammond, P. and Fothergill, L. K. (1994) Quantification of excitatory receptive fields of complex neurones in cat striate cortex. Exp. Brain Res., 99, 170-174.

Hubel, D. H. and Wiesel, T. N. (1965) Binocular interaction in striate cortex of kittens reared with artificial squint. J. Neurophysiol., 28, 1041-1059.

Kalil, R. E., Spear, P. D. and Langsetmo, A. (1984) Response properties of striate cortex neurons in cats raised with divergent or convergent strabismus. J. Neurophysiol., 52, 514-537.

Kanizsa, G. (1979) Organization in Vision: Essays on Gestalt Perception. Praeger, New York.

Kapadia, M. K., Ito, M., Gilbert, C. D. and Westheimer, G. (1995) Improvement 
in visual sensitivity by changes in local context: parallel studies in human observers and in V1 of alert monkeys. Neuron, 15, 843-856.

Katz, L. C. and Iarovici, D. M. (1990) Green fluorescent latex microspheres: a new retrograde tracer. Neurosci., 34, 511-520.

Katz, L. C., Burkhalter, A. and Dreyer, W. J. (1984) Fluorescent latex microspheres as a retrograde neuronal marker for in vivo and in vitro studies of visual cortex. Nature, 310, 498-500.

Kisvárday, Z. F. and Eysel, U. T. (1992) Cellular organization of reciprocal patchy networks in layer III of cat visual cortex (area 17). Neuroscience, 46, 275-286.

Löwel, S. and Singer, W. (1992) Selection of intrinsic horizontal connections in the visual cortex by correlated neuronal activity. Science, 255, 209-212.

Luhmann, H. J., Singer, W. and Martinez-Millan, L. (1991) Horizontal interactions in cat striate cortex: I. Anatomical substrate and postnatal development. Eur. J. Neurosci., 2, 358-368.

Malach, R., Amir, Y., Harel, M. and Grinvald, A. (1993) Relationship between intrinsic connections and functional architecture revealed by optical imaging and in vivo targeted biocytin injections in primate striate cortex. Proc. Natl Acad. Sci. USA, 90, 10469-10473.

Martin, K. A. C. and Whitteridge, D. (1984) Form, function and intracortical projections of spiny neurons in the striate visual cortex of the cat. $J$. Physiol. (Lond.), 353, 463-504.

Mitchison, G. and Crick, F. (1982) Long axons within the striate cortex: their distribution, orientation and pattern of connections. Proc. Natl Acad. Sci. USA, 79, 3661-3665.

Munk, M. H. J., Nowak, L. G., Nelson, J. I. and Bullier, J. (1995) The structural basis of cortical synchronization. 2. Effects of cortical lesions. $J$. Neurophysiol., 74, 2401-2414.

Nelson, J. I. and Frost, B. J. (1985) Intracortical facilitation among co- oriented, co-axially aligned simple cells in cat striate cortex. Exp. Brain Res., 61, 54-61.

Polat, U. and Sagi, D. (1993) Lateral interactions between spatial channels: suppression and facilitation revealed by lateral masking experiments. Vision Res., 33, 993-999.

Polat, U. and Sagi, D. (1994) The architecture of perceptual spatial interactions. Vision Res., 34, 73-78.

Rockland, K. S. and Lund, J. S. (1982) Widespread periodic intrinsic connections in the tree shrew visual cortex. Science, 215, 1532-1534.

Schmidt, K. E., Kim, D.-S., Singer, W., Bonhoeffer, T. and Löwel, S. (1994) Optical imaging of the orientation map and horizontal intrinsic connections in strabismic cats. Soc. Neurasci. Abstr., 20, 313.

Sincich, L. and Blasdel, G. G. (1995) Lateral connections and orientation preference in layers II/III of squirrel monkey striate cortex. Soc. Neurosci. Abstr, 21, 393

Singer, W. (1995) Development and plasticity of cortical processing architectures. Science, 279, 758-764.

Singer, W., Rauschecker, J. and von Grünau, M. (1979) Squint affects striate cortex cells encoding horizontal image movements. Brain Res., 170, $182-186$.

Sireteanu, R. and Best, J. (1992) Squint-induced modification of visual receptive fields in the lateral suprasylvian cortex of the cat: binocular interaction, vertical effect and anomalous correspondence. Eur. J. Neurosci. $4,235-242$.

Sireteanu, R. and Singer, W. (1980) The 'vertical effect' in human squint amblyopia. Exp. Brain Res., 40, 354-357.

Ts'o, D. and Gilbert, C. D. (1989) The organization of chromatic and spatial interactions in the primate striate cortex. J. Neurosci. 8, 1712-1727.

Ts'o, D., Gilbert, C. D. and Wiesel, T. N. (1986) Relationships between horizontal interactions and functional architecture in cat striate cortex as revealed by cross-correlation analysis. J. Neurosci., 6, 1160-1170. 